\title{
Terpenbiosynthese
}

\section{Neue Klasse von Methyltransferasen mit Zyklisierungsaktivität}

\author{
BIRGIT PIECHULLA ${ }^{1}$, NANCY MAGNUS ${ }^{1}$, MARIE CHANTAL LEMFACK ${ }^{1}$, \\ STEPHAN VON REUSS ${ }^{2}$ \\ ${ }^{1}$ INSTITUT FÜR BIOWISSENSCHAFTEN, UNIVERSITÄT ROSTOCK \\ 2 INSTITUT FÜR CHEMIE, UNIVERSITÄT NEUCHÂTEL, SCHWEIZ
}

\section{Microorganisms release small volatile metabolites with unique struc- tures, e. g. the polymethylated homosesquiterpene sodorifen from Serratia plymuthica. Two unusual enzymes with novel features are involved in its biosynthesis, a $C$-methyltransferase with cyclization activity and a terpene synthase that accepts a non-canonical mono- cyclic $C_{16}$ substrate. The novel class of methyltransferases represents an alternative route that enlarges terpene diversity.}

DOI: $10.1007 / \mathrm{s} 12268-021-1506-8$

(C) Die Autoren 2021

Mikroorganismen produzieren Naturstoffe (Sekundärmetabolite), die beispielsweise als Antibiotika eingesetzt werden. Neben diesen meist hochmolekularen Substanzen bilden Bakterien und Pilze aber auch eine Vielzahl von kleinen Metaboliten, die sehr häufig flüchtig sind und Duftstoffe repräsentieren. Einige dieser Metabolite sind seit langem bekannt und werden als Aromen und Konservierungsstoffe in der Lebensmittelproduktion eingesetzt, wie die Produkte der Milchsäuregärung (Sauerkraut, Wurst, Käse) und der Ethanolgärung (Wein, Bier). Auch der erdige Geruch des Geosmins aus Streptomyceten ist markant und wird häufig mit „Wald“ assoziiert, obwohl dieser auch für verunreinigtes Wasser charakteristisch ist.

In den vergangenen 15 Jahren erlangten diese microbial volatile organic compounds (mVOCs) neue Aufmerksamkeit, da durch mVOCs ausgelöste biologische und ökologische Effekte in Mikrobiomen und Holobionten beobachtet wurden. Bisher sind etwa 2.000 mVOCs bekannt, von denen bislang für rund 100 Verbindungen biologische Funktionen beschrieben wurden [1]. Diese reichen von Wachstumsförderungen, Änderungen der Physiologie bis zu Abwehrmechanismen in Pflanzen und Pilzen. mVOCs verbessern in Vertebraten und Invertebraten die Immunantworten und induzieren die Eiablage. Zudem wurde sowohl eine Inhibition des
Lymphozytenwachstums und der Cytokinproduktion beobachtet als auch eine Reduktion der Beweglichkeit der Nematoden und eine nematizide Wirkung. mVOCs beeinflussen auch die Etablierung von Mikrobiomen: Sie hemmen beispielsweise die Biofilmbildung, stimulieren oder hemmen das Wachstum sowie die Sporenbildung und -keimung bei Pilzen. Bisher wurden vor allem phänotypische Veränderungen untersucht, während über die molekularen Mechanismen wenig bekannt ist.

Die mVOCs von Bakterien und Pilzen sind in der mVOC-Datenbank [2] zusammengefasst. Darunter befinden sich Verbindungen mit einfachen Strukturen, wie Butandiol, Oktanol, Indol, Essigsäure und ähnliche, allerdings auch solche mit sehr komplexen, teilweise bisher unbekannten Strukturen, deren Biosynthesen nicht immer leicht abzuleiten sind, wie beispielsweise das Sodorifen (Abb. 1).

\section{Serratia plymuthica 4Rx13 produziert Sodorifen}

Serratia-Spezies gehören zu den Enterobacteriaceae und kommen ubiquitär vor. Sie sind anders als Streptomyceten nicht für die Biosynthese von außergewöhnlichen Sekundärmetaboliten bekannt. Jedoch rückte Serratia plymuthica 4Rx13, ursprünglich aus der Rhizosphäre von Raps in der Umgebung von
Rostock isoliert, in das Zentrum der Forschung von neuen mVOCs. Es handelt sich um einen außergewöhnlichen Naturstoff $\left(\mathrm{C}_{16} \mathrm{H}_{26}\right.$, Sodorifen), detektierbar im headspace (Luftraum) über der Bakterienkultur. Die Strukturaufklärung ergab einen bizyklischen Kohlenwasserstoff, in dem jedes Gerüstatom mit einer Methylgruppe verknüpft ist [3]. Anders als erwartet, markiert isotopenmarkiertes Methionin nur eine Methylgruppe, was den Schluss zuließ, dass die anderen Methylgruppen durch intramolekulare Umlagerung entstehen müssten. Die Aufklärung der Biosynthese von Sodorifen ergab, dass dieser Naturstoff ein Homosesquiterpen ist, das von zwei Enzymen mit neuen Eigenschaften gebildet wird [4].

Der Terpenstoffwechsel von Bakterien ist im Vergleich zu Pflanzen wenig untersucht. Obwohl mehr als $10^{6}$ Bakterienspezies und über 30.000 Genome beschrieben wurden, sind derzeit weniger als 100 bakterielle Terpensynthasen, meistens Sesquiterpensynthasen, bekannt. Etwa 90 Prozent der bisher isolierten Terpensynthasen stammen aus Streptomyceten. Die Terpensynthase aus Serratia plymuthica ist somit ein Unikat. Generell sind Terpensynthasen schwer in den Bakteriengenomen zu lokalisieren; bisher konnten nur rund 250 Gene in verschiedenen Genomuntersuchungen aufgespürt werden.

In der Tat unterscheiden sich die bisher gefundenen bakteriellen TerpensynthaseGene deutlich von den pflanzlichen Konterfei-Genen. Statt 550 Aminosäuren sind sie nur etwa 350 Aminosäuren lang, sie sind nicht wie die Enzyme der Pflanzen aus alpha-, beta- und gamma-Domänen aufgebaut, sondern nur aus alpha-, alpha-alphaoder beta-gamma-Domänen. Während die pflanzlichen Terpensynthasen mehrere konservierte Sequenzmotive enthalten, ist in den bakteriellen Genen nur das aspartatreiche Motiv, das für die Bindung der Metallionen beziehungsweise des Substrats verantwortlich ist, konserviert. Obwohl offensichtlich der Aufbau der Terpensynthase aus Serratia einfacher als die in Pflanzen ist, ist dieses 
<smiles>CC(C)=CCCC(C)=CCOc1ccccc1</smiles>

GPP

B

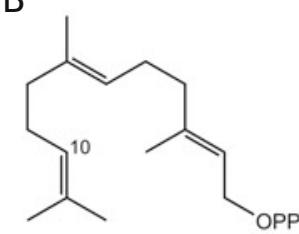

FPP
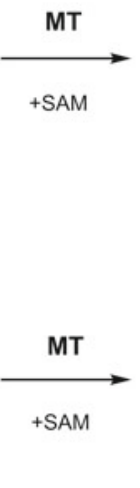

$$
\text { Pre-Sodorifen-PP }
$$

2-Methyl-GPP
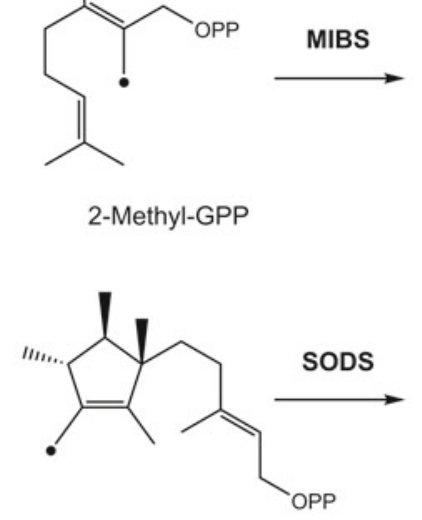

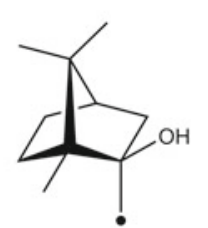

2-Methyl-Isoborneol

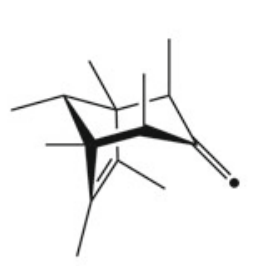

Sodorifen

$\Delta$ Abb. 1: Methylierungen von Prenyl-Pyrophosphaten. A, GPP-Methyltransferase methyliert Geranylpyrophosphat (GPP) an Position 2; es entsteht 2-Methyl-GPP, was wiederum das Substrat für die Methyl-Isoborneol-Synthase (MIBS) ist. B, FPP wird zunächst an Position 10 methyliert und nachfolgend zu Pre-Sodorifen-Pyrophosphat zyklisiert; beide Reaktionsschritte werden durch die neue Klasse der Prenyl-Pyrophosphat-C-Methyltransferasen katalysiert. Pre-Sodorifen-Pyrophosphat ist ein neues Substrat für Terpensynthasen, hier speziell für die Sodorifen-Terpensynthase (SODS).

Enzym dennoch in der Lage, die ungewöhnlich komplexe Produktstruktur des Sodorifens zu generieren. Wie dies möglich ist, wurde deutlich, nachdem das SodorifenCluster im S. plymuthica-Genom entschlüsselt wurde [4].

\section{Neue Methyltransferase-Aktivität identifiziert}

Das Sodorifen-Cluster von S. plymuthica 4Rx13 enthält vier Gene, die für eine Isomerase, eine Desoxy-Xylulose-PhosphatSynthase, eine Methyltransferase und eine Terpensynthase codieren. Während die beiden erstgenannten Enzyme für die Bereitstellung des Isopentenylpyrophosphats verantwortlich sind, produzieren die Methyltransferase und die Terpensynthase Sodorifen. Dies untermauerten $u$. a. Untersuchungen mit Knockout-Mutanten und in vitro-DoppelEnzymassays mit heterolog überexprimierten Enzymen. Die S-Adenosyl-Methionin abhängige Farnesylpyrophosphat(FPP)Methyltransferase methyliert die Position 10 des FPP und bildet in einem bisher einzigartigen Mechanismus ein monozyklisches Produkt, das Pre-Sodorifen Pyrophosphat (Abb. 1). Dieses zyklische Intermediat ist nun das Substrat für die nachfolgende Sodorifen-Terpensynthase (SODS). Bisher waren nur die aliphatischen Prenylpyrophosphate GPP, FPP, NPP, GGPP und entsprechende Stereoisomere als Substrate für Terpensynthasen bekannt. Die Sodorifen-Synthase von
S. plymuthica ist somit die erste Terpensynthase, die ein zyklisches (nicht-kanonisches) Substrat verwendet, dieses intramolekular umlagert, um das bizyklische Produkt Sodorifen zu bilden. Die SODS akzeptiert wiederum kein azyklisches FPP.

Die Biosynthese der ungewöhnlichen Struktur des Sodorifens beruht auf dem Einsatz von Enzymen mit ungewöhnlichen Fähigkeiten und Aktivitäten unter Nutzung jeweils ungewöhnlicher Substrate (Abb. 1). Insbesondere die FPP-Methyltransferase ist dabei hervorzuheben, denn sie repräsentiert eine neue Klasse von „zyklisierenden C-Methyltransferasen“. Untermauert wird diese Hypothese dadurch, dass bisher keine zufriedenstellenden Methyltransferase-Templates für die in silico-Analyse gefunden wurden und Sequenzidentitäten mit ,ähnlichen“ Enzymen nur bei 17-18 Prozent liegen.

Die kanonische Terpenbiosynthese basiert auf der sukzessiven Verlängerung mit $\mathrm{C}_{5}$-Einheiten, welche die charakteristischen Terpenklassen, Monoterpene $\left(\mathrm{C}_{10}\right)$, Sesquiterpene $\left(\mathrm{C}_{15}\right)$, Diterpene $\left(\mathrm{C}_{20}\right)$ usw. ergeben. Die kanonischen Substrate GPP, FPP, GGPP usw. liefern azyklische Produkte oder werden durch spezifische Terpensynthasen zyklisiert. Produktdiversität wird durch Multiprodukt-Terpensynthasen sowie durch nachgeschaltete dekorierende Enzyme (Cytochrom P450, Dehydrogenasen, Methyltransferasen usw.) erreicht. Während das pflanzliche Homoterpen TMTT vom $\mathrm{C}_{20}(\mathrm{E}, \mathrm{E})$-Gera- nyllinalool abstammt [5], wird $\mathrm{C}_{16}$-Sodorifen von S. plymuthica durch Methylierung des kanonischen $\mathrm{C}_{15}$ FPP generiert. Die $S$. $p$.FPPMT ist die erste Methyltransferase, die gleichzeitig methyliert und zyklisiert [4]. Im Gegensatz dazu sind einige C-methylierende GPPMTs aus Streptomyceten bekannt [6, 7, 8]. Diese Enzyme methylieren GPP an Position 2; es entsteht 2-Methyl-GPP, das die IsoborneolSynthase zu 2-Methyl-Isoborneol umsetzt. Diese methylierten Substrate werden von promiskuitiven Terpensynthasen genutzt, um neuartige C11-Homoterpenoide zu bilden [9, 10, 11]. IPP kann auch methyliert werden, um die Produktdiversität zu erhöhen [12] und in Lepidoptera führt die Fusion von Propionyl-CoA mit Acetyl-CoA zur Bildung von $\mathrm{C}_{16}$-Terpenen [13].

Es wird deutlich, dass die neue Klasse der pPPMTs in Bakterien eine alternative Route eröffnet, um das Substratangebot für die Biosynthese von Terpenen zu erweitern, und damit zur Terpendiversität beiträgt. Bisher wurden diese methylierten pPP-Substrate nur in Bakterien gefunden. Diese Präsenz in Bakterien wirft jedoch die Frage bezüglich des Ursprungs dieses Weges und der Co-Evolution der Gene auf. Es bleibt spannend für die Zukunft!

\section{Literatur}

[1] Piechulla B, Lemfack MC, Kai M (2017) Effects of discrete bioactive microbial volatiles on plants and fungi. Plant Cell Environ 40: 2042-2067

[2] Lemfack MC, Gohlke B, Toguem SMT et al. (2018) mVOC 2.0: a database of microbial volatiles. Nucleic Acids Res 46(D1): D1261-D1265

[3] von Reuss S, Kai M, Piechulla B, Francke W (2010) Octamethylbicyclo(3.2.1)octadienes from Serratia odorifera. Angew Chem Int Ed 49: 2009-2010

[4] von Reuss S, Domik D, Lemfack MC et al. (2018) Sodorifen biosynthesis in the rhizobacterium Serratia plymuthica 4Rx13 involves methylation and cyclization of MEPderived farnesyl pyrophosphate by a SAM-dependent C-methyltransferase. J Am Chem Soc 140: 11855-11862 [5] Boland W, Gäbler A, Gilber M, Feng Z (1998) Biosynthesis of C11 and C16 homoterpenes in higher plants: Stereochemistry of the C-C bond cleavage reaction. Tetrahedron 54 14725-14736

[6] Wang CM, Cane DE (2008) Biochemistry and molecular genetics of the biosynthesis of the earthy odorant methylisoborneol in Streptomyces coelicolor. J Am Chem Soc 130: 8908-8909

[7] Komatsu M, Tsuda M, Omura S et al. (2008) Identification and functional analysis of genes controlling biosynthesis of 2-methylisoborneol. Proc Natl Acad Sci USA 105: 7422-7427 [8] Köksal M, Chou WK, Cane DE, Christianson DW (2012) Structure of geranyl diphosphate C-methyltransferase from Streptomyces coelicolor and implications for the mechanism of isoprenoid modification. Biochemistry 51: 3003-3010

[9] Kschowak MJ, Wortmann H, Dickschat JS et al. (2018) Heterologous expression of 2-methylisoborneol / 2 methylenebornane biosynthesis genes in Escherichia coli yields novel C11-terpenes. PLoS One 13: e0196082

[10] Ignea C, Pontini M, Motawia MS et al. (2018) Synthesis of 11-carbon terpenoids in yeast using protein and metabolic engineering. Nat Chem Biol 14: 1090-1098

[11] Duell ER, D'Agostino PM, Shapiro N et al. (2019) Direct pathway cloning of the sodorifen biosynthetic gene cluster and recombinant generation of its product in E. coli. Microb Cell Fact 18: 32 
[12] Drummond L, Kschowak MJ, Breitenbach J et al. (2019) Expanding the isoprenoid building block repertoire with an IPP methyltransferase from Streptomyces monomycini. ACS Synth Biol 8: 1303-1313

[13] Eiben CB, deRond T, Bloszies C et al. (2019) Mevalonate pathway promiscuity enables noncanonical terpene production. ACS Synth Biol 8: 2238-2247

Funding note: Open Access funding enabled and organized by Projekt DEAL. Open Access: Dieser Artikel wird unter der Creative Commons Namensnennun 4.0 International Lizenz veroffentlicht, welche die Nutzung, Vervielfaltigung, erlabt, ordnungs a enthaltenen Bilder und sonstiges Drittmaterial unterliegen ebenfalls der genannten Creative Commons Lizenz, sofern sich aus der Abbildungslegende nichts anderes ergibt. Sofern das betreffende Material nicht unter der genannten Creative Commons Lizenz steht und die betreffende Handlung nicht nach gesetzlichen Vorschriften erlaubt ist, ist für die oben aufgeführten Weiterverwendungen des Materials die Eimilligung des jeweiligen Rechteinhabers einzuholen. Weitere Details zur Lizenz entnehmen Sie bitte der enses/by/4.0/deed.de.

\section{Korrespondenzadresse:}

Prof. Dr. Birgit Piechulla

Institut für Biowissenschaften

Universität Rostock

Albert-Einstein-Straße 3

D-18059 Rostock

birgit.piechulla@uni-rostock.de

www.biochemie.uni-rostock.de

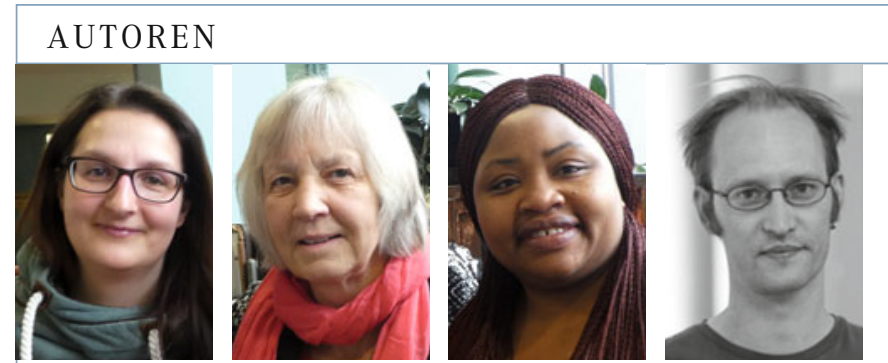

Nancy Magnus, Birgit Piechulla, Marie Chantal Lemfack und Stephan von Reuss (v. I. n. r.)

\section{Birgit Piechulla}

1975-1980 Biologiestudium an den Universitäten Oldenburg und Göttingen. 1983 Promotion an der Universität Göttingen. 1984-1986 Postdoc an der UC Berkeley, CA, USA. 1987-1992 Habilitation Universität Göttingen. Seit 1996 C4-Professur am Lehrstuhl für Biochemie an der Universität Rostock.

Marie Chantal Lemfack

2001-2007 Biochemiestudium an der Universität Dschang, Kamerun. 2016 Promotion an der Universität Rostock. Seit 2016 Postdoc am Lehrstuhl für Biochemie an der Universität Rostock.
Nancy Magnus

2006-2012 Biologiestudium an der Universität Rostock, dort 2018 Promotion. Seit 2018 Postdoc am Lehrstuhl für Biochemie an der Universität Rostock.

\section{Stephan von Reuss}

2004 MSc in Chemie an der Universität Hamburg. 2009 Promotion an der Universität Hamburg. 2009-2012 Postdoc an der Cornell University, NY, USA und 2012-2016 am Max-Planck-Institut für Chemische Ökologie in Jena. Seit 2016 Professor an der Universiät Neuchâtel, Schweiz.

\section{Hier steht} eine Anzeige. 